\title{
The Lipase-catalyzed Synthesis of Ethyl Ferulate
}

\author{
Qiannan Wang ${ }^{1, a}$, Wei Zhang ${ }^{1, b}$ and Jiaying Xin ${ }^{1, c^{*}}$ \\ ${ }^{1}$ Key Laboratory for Food Science and Engineering, Harbin University of Commerce, China \\ a543688467@qq.com, b1562314138@qq.com, cxinjiayingvip@163.com
}

\section{Keywords: Lipase; Ethyl ferulate; Biocatalytic synthesis; Ferulic acid}

\begin{abstract}
Selective biocatalytic synthesis is an important part of the third generation of biotechnology, is the forefront of international scientific and technological development. Utilizing enzyme catalyzed reaction, many complex substances can be synthesized. Biocatalysis have strict regional selectivity and enantioselectivity. Ferulic acid is naturally safe radical quencher and anti-oxidation agent. In the cosmetics industry, ferulic acid is considered to be the best natural sunscreen, also used as skin whitening agents. Ferulic acid and its derivatives are widely used in the pharmaceutical industry, the application in the food industry are also increasing. This paper studied the immobilized lipase-catalyzed synthesis of ethyl ferulate in organic medium. Lipase from Mucor sp.M-2 was immobilized with diatomite powder as the carrier by absorption and was used as biocatalyst. The reaction medium were compared for biocatalytic synthesis of ethyl ferulate. The optimum solvent is tert-butyl alcohol . The opitimization of reaction conditions was obtained. In the $4 \mathrm{ml}$ of tert butyl alcohol, when the molar ratio of acid to alcohol is $1.5: 1$, the temperature is 45 degrees centigrade, a yield of $9.9 \%$ for ethyl ferulate can be obtained after $120 \mathrm{~h}$.
\end{abstract}

\section{Introduction}

Ferulic acid (4-hydroxy-3-methoxy-cinnamic acid) is a phenolic compound in the presence of higher plants, usually found in corn, rice bran and wheat bran cell wall and insoluble fibers [1]. In recent years, ferulic acid has become a kind of biochemical products in the pharmaceutical, food, cosmetics and other fields and has more and more widely used. Ferulic acid has anti-inflammatory, analgesic, anti thrombosis, anti ultraviolet radiation, anti free radical generation, regulate endocrine, and regulate the body's immune function and so on [2]. Ferulic acid, a most abundant derivative of cinnamic acid is found in higher plants. It is ester linked to cell wall constituents especially to arabinoxylans and lignin's.[3] Ferulic acid is a natural and safe free radical quenching agent and antioxidant, which has been paid much attention in the study of anti-aging. It has maximum UV absorption at $322 \mathrm{~nm}$ which falls between the UVB and UVA region and hence can be used as a potential UV-absorbing substance for skin protect, also used as a skin lightening agent and a cell differentiation promoter. It used in the clinical treatment of coronary heart disease, cerebrovascular disease, vasculitis, neutropenia and thrombocytopenia and other diseases. In the food industry it is mainly used as an antioxidant, but its hydrophilic limits its application in oil-soluble foods [4].

However, ferulic acid has low solubility in both aqueous and organic phases and is also unstable at high temperature where it undergoes oxidation.The study found that after chemical esterification modification, ferulic acid has a stronger physiological activity and antioxidant activity [5]. But the chemical synthesis can bring toxic substances, side-product, serious environmental pollution, poor selectivity, high energy consumption. The severe reaction conditions can damage the structure of natural compounds. Natural, nutrition, and multi-purpose is the goal for the development of the food additives industry. The international food additive requirements are more natural, fresh and enhance food quality during storage stability; no or little use of chemical synthetic additives [6].Therefore chemical synthesis does not comply with international food requirements additives [7]. Food additive made by biotechnology is known as a food additive "green manufacturing". it is one of the main areas of core technology of modern food industry. To avoid the drawbacks of chemical synthesis ethyl 
ferulate, ethyl ferulate was enzymatic synthesized in organic medium based on the non-aqueous medium biocatalysis [8].

\section{Materials and Methods}

Chemicals and Materials. Ethyl ferulate standard and ferulic acid are obtained from Sinopharm Chemical Reagent Co. Ltd. Other chemicals were of analytical reagent grade and used without further purification. All stock solution were prepared daily with distilled water [9].

The immobilized lipase was obtained from Mucor sp.M-2 by fermentation and then immobilization.

The conditions for the production of lipase was as follows: $4.0 \mathrm{~g}$ soybean powder, $0.1 \mathrm{~g}(\mathrm{NH} 4) 2 \mathrm{SO} 4,0.5 \mathrm{~g}$ sucrose, $1 \mathrm{ml}$ emulsified-olive, $0.015 \mathrm{~g} \mathrm{MgSO} 4$, nature $\mathrm{pH}$. The optimum fermentation temperature and the optimum agitation speed were 28 degrees centigrade and $250 \mathrm{r} / \mathrm{min}$, respectively. After $74 \mathrm{~h}$ incubation with the optimum medium under the condition of $250 \mathrm{r} / \mathrm{min}$ and 28 degrees centigrade, a maximum lipase yield was obtained.

The lipase was immobilizd with diatomite powder as the carrier by absorption. The activity of immobilized lipase was $2500 \mathrm{U} / \mathrm{g}$.

Product Analysis and Detection. The standard curve of ethyl ferulate was drawn as below: $22.2 \mathrm{mg}$ of Ethyl ferulate standard sample were weighed accurately and turned it into a $100 \mathrm{ml}$ volumetric flask, then add methanol dissolved and diluted to the scale

$1-10 \mathrm{uL}$ of the samples were pointed on the same silica gel GF TLC plate, with the developing solvent benzene: ether and dichloromethane: n-hexane =1:1:2:6 (V / V). The standard curve was obtained with ethyl ferulate standard concentration as the abscissa and spots peak area integral value as ordinate [10].

Effect of Organic Solvents on Esterification Reaction. Five $25 \mathrm{ml}$ flask were prepared. 2.0 mmol of ethanol were added. After the removal of water by molecular sieve, adding $1.0 \mathrm{mmol}$ ferulic acid, $0.5 \mathrm{~g}$ immobilized lipase, $150 \mathrm{r} / \mathrm{min}$ air bath oscillation reaction at a temperature of 35 degrees centigrade and 150r/min air bath oscillation reaction $96 \mathrm{~h}$.

The Effect of Organic Solvents on the Esterification Reaction. 1.0mmol ferulic acid, $2.0 \mathrm{mmol}$ of ethanol and molecular sieves were added into $25 \mathrm{ml}$ flask. Different organic solvents were used respectively., $0.5 \mathrm{~g}$ of immobilized lipase were added to catalyze the reaction at a temperature of 35 degrees centigrade and 150r/min for $96 \mathrm{~h}$.

The Effect of Substrate Molar Ratio on the Esterification Reaction. Ethanol and ferulic acid and molecular sieves were added into $25 \mathrm{ml}$ flask with different molar ratio of ethanol to ferulic acid. tert butyl alcohol was used as reaction medium, After removal of water by molecular sieve, $0.5 \mathrm{~g}$ of the immobilized enzyme was used to catalyze the reaction at 35 degrees centigrade and $150 \mathrm{r} / \mathrm{min}$ for $96 h$.

The Effect of Temperature on Esterification Reaction. $1.0 \mathrm{mmol}$ ferulic acid, $1.5 \mathrm{mmol}$ of ethanol, $4 \mathrm{ml}$ of Tert butyl alcohol and molecular sieves were added into $25 \mathrm{ml}$ flask. After removal of water by molecular sieve, $0.4 \mathrm{~g}$ of the immobilized enzyme was used to catalyze the reaction at different temperature and $150 \mathrm{r} / \mathrm{min}$ for $96 \mathrm{~h}$.

The Effect of Reaction Time on Esterification Reaction. $1.0 \mathrm{mmol}$ ferulic acid, $1.5 \mathrm{mmol}$ of ethanol, $4 \mathrm{ml}$ of Tert butyl alcohol and molecular sieves were added into $25 \mathrm{ml}$ flask. After removal of water by molecular sieve, $0.4 \mathrm{~g}$ of the immobilized enzyme was used to catalyze the reaction at 45 degrees centigrade and 150r/min for different reaction time.

\section{Results and Discussion}

Table1 showed the effect of organic solvents on the esterification reaction. The results showed that dimethyl sulfoxide, acetonitrile, tetrahydrofuran has better solubility for ferulic acid. However, these organic solvent but very large, result in the rapid inactivation of lipase and no catalytic reaction 
happened, Toluene is good for the stability of the lipase but has poor solubility for ferulic acid, Tert butyl alcohol with $\log \mathrm{P}$ value of 0.8 , has good for the stability of the lipase and solubility for ferulic acid, So tert butyl alcohol can ensuring the substrate have good solubility and the enzyme has good stability and ferulic acid yield can reach to $4.2 \%$. At the same time, because of the large steric hindrance of tert-butyl alcohol, the esterification reaction of ferulic acid with the presence of tert-butyl alcohol would not occur, so it was determined that tert butyl alcohol as an ideal reaction medium.

Table 1 The effect of organic solvents on the esterification reaction

\begin{tabular}{lll}
\hline Organic solvent & $\log \mathrm{P}$ & $\begin{array}{c}\text { Production rate of 96 hours } \\
(\%)\end{array}$ \\
\hline Dimethyl sulfoxide & -1.3 & 0.0 \\
Acetonitrile & -0.33 & 0.0 \\
Tetrahydrofuran & 0.49 & 0.0 \\
Tert-butanol & 0.80 & 4.2 \\
Toluene & 2.5 & 1.1 \\
\hline
\end{tabular}

As shown in Fig. 1, in the reaction system, from 2 to $8 \mathrm{ml}$ of tert-butyl alcohol was added respectively and the addition amount of $4 \mathrm{ml}$ has the highest conversion of ferulic acid ethyl ester. After the addition amount of tert-butyl alcohol was more than $4 \mathrm{ml}$, the decreasing of the conversion has been found. Enzyme substrate has a certain impact.

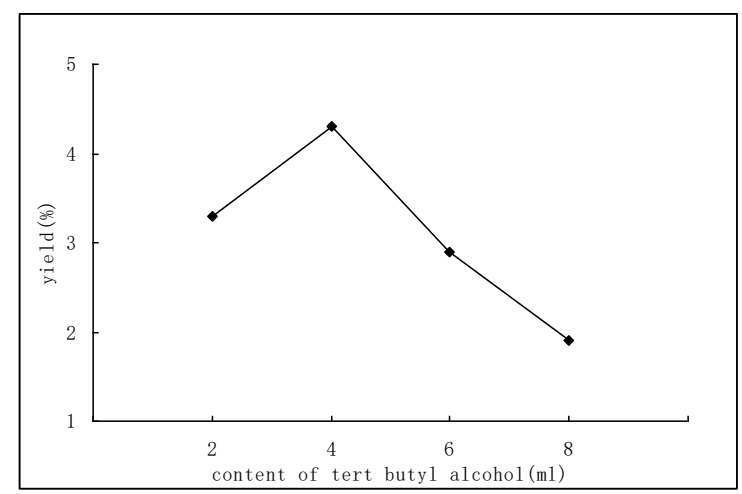

Figure 1. The addition amount of Tert butyl alcohol on the yield of ethyl ferulate

As shown in Fig. 2. with the increasing of molar ratio of ethanol to ferulic acid, the yield of ethyl ferulate increased significantly The potential reason may be that the esterification reaction is a reversible reaction, increasing the amount of ethanol, the reaction equilibrium in favor of moving towards the direction of ferulic acid ethyl ester generated, thereby increasing the yield of ferulic acid ethyl ester. When the molar ratio of ethanol to ferulic acid reached 1.5:1, the yield reached maximum. From the above results, the optimum molar ratio of ferulic acid to ethanol is $1.5: 1$. 


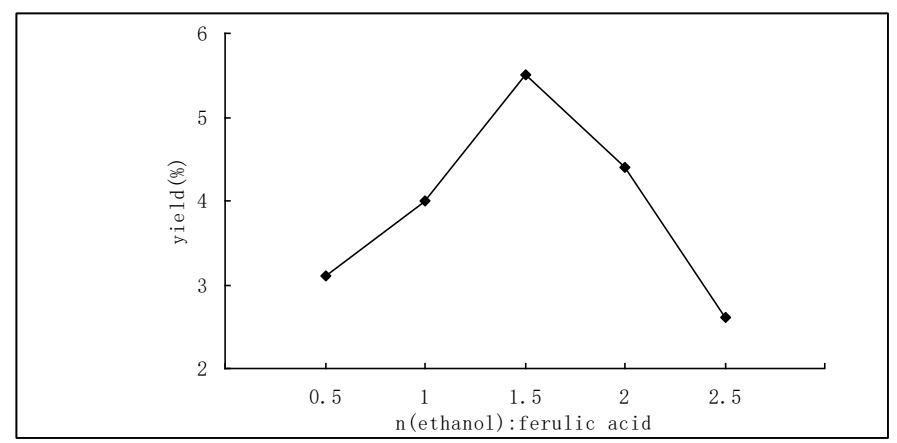

Figure 2. The effect of substrate ratio on the yield of ethyl ferulate

The reaction temperature is the important factors which decided the yield of enzymatic reaction. As shown in Fig. 3. The influence of reaction temperature on the yield was studied. Fixed other reaction conditions, the enzymatic esterification of ferulic acid with alcohol were carried out at different temperatures for $96 \mathrm{~h}$. When the enzymatic reaction temperature is 30 degrees centigrade, yield of the esterification was low. With the temperature gradually increased, the yield of the esterification was gradually increased, when the temperature reached 45 degrees centigrade. The highest yield of $7.4 \%$ was obtained. With further increase of temperature, the yield of the reaction began to decline rapidly. This showed that the stability of immobilized lipase in tert butanol solution is good and can play a higher catalytic activity at higher temperatures. When the temperature was more than 45 degrees centigrade, due to the inactivation of the enzyme, the yield declined rapidly, so the temperature of the reaction system is 45 degrees centigrade.

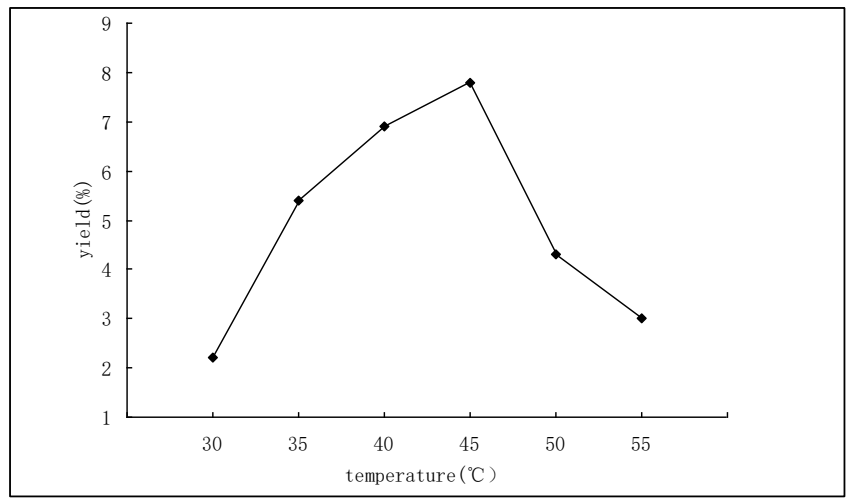

Figure 3. The effect of the reaction tenperature on the yield of ethyl ferulate

As shown in Fig. 4, with the increase of reaction time, the yield increased gradually after reaction of $120 \mathrm{~h}$, esterification yield no longer increased. This may be due to the production of water in the process of esterification reaction, which inhibits the esterification reaction. Therefore the best reaction time was $120 \mathrm{~h}$.

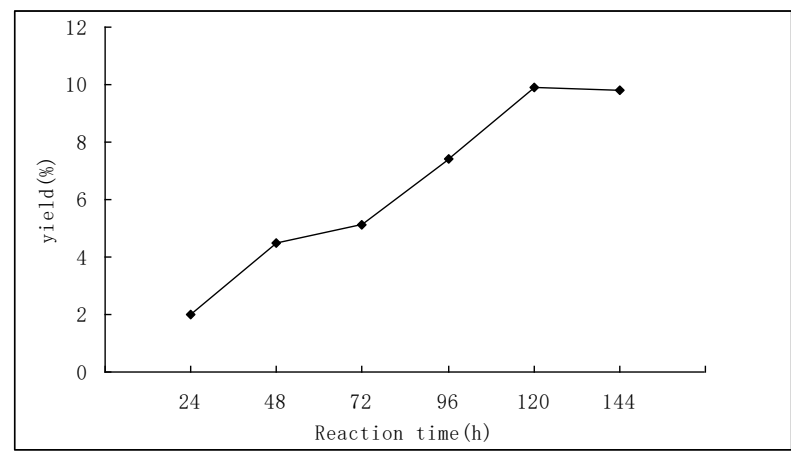

Figure 4. The effect of reaction time on the yield of ethyl ferulate 


\section{Summary}

Lipase-catalyzed esterification of ferulic acid with ethanol in organic solvent was studied. The optimum solvent was tert-butyl alcohol .The opitimization of reaction conditions was obtained. In the $4 \mathrm{ml}$ of tert butyl alcohol, when the molar ratio of alcohol to ferulic acid is $1.5: 1$, the temperature is 45 degrees centigrade, a yield of $9.9 \%$ for ethyl ferulate can be obtained after $120 \mathrm{~h}$.

\section{References}

[1] JIANG Jian-jun, WANG Lin-shan. The application technology of food additive[M]. Beijing: Science Press, 2004.

[2] Zhang Yueling, Weichang Mei, Wang Jintang ferulic acid synthesis and molecular transformation [J]. Huaiyin Teachers College, 2003,2 (1): 53-43.

[3] Murakami, A., Nakamura, Y., Koshimizu, K., Takahashi, D., Matsumoto, K., Hagihara, K., Taniguchi, H., Nomura, E., Hosoda, A., Tsuno, T., Maruta, Y., Kim, H.W., Kawabata, K., Ohigashi, H., 2002. FA15, a hydrophobic derivative of ferulic acid, suppresses inflammatory responses and skin tumor promotion: comparison with ferulic acid. Cancer Lett. 180, 121-129.

[4] Saija, A., Tomaino, A., Cascio, R.L., Trombetta, D., Proteggente, A., Pasquale, A.D., Uccella, N., Bonina, F., 1999. Ferulic and caffeic acids as potential protective agents against photooxidative skin damage. J. Sci. Food Agric. 79, 476-480.

[5] Sun, S., Shan, L., Jin, Q., Liu, Y., Wang, X., 2007. Solvent free synthesis of glyceryl ferulate using a commercial microbial lipase. Biotechnol. Lett. 29, 945-949.

[6] Fang X,Shima M.Suppressive effect of alkyl ferulate on the oxidation of linoleic acid[J].Biosci Biotechnol Biochem,2006,70:457-461.

[7] Stamatis H,Sereti V,Kolisis F N.Enzymatic synthesis of hydrophilic and hydrophobic derivatives of natural phenolic acid in organic media [J].Mol Catal B:Enzymatic,2001(11):323-328.

[8] Dandavate, V., Jinjala, J., Kehria, H., Madamwar, D., 2009. Production partial purification and characterization of organic solvent tolerant lipases from Burkholderia multivorans V2 and its applications for ester synthesis. Bioresour. Technol. 100, 3374-3381.

[9] Kumar, S., Ola, R.P., Kaushal, R., Kanwar, S.S., Gupta, R., 2006. Thermostability and esterification studies of a polyethylene-immobilized lipase from Bacillus coagulans BTS-3. J. Appl. Polym. Sci. 102, 3986-3993.

[10]Tsuchiyama, M., Sakamoto, T., Fujita, T., Murata, S., Kawasaki, S., 2006. Esterification of ferulic acid with polyols using a ferulic acid esterase from Aspergillus niger. Biochim. Biophys. Acta Gen. Subj. 1760, 1071-1079. 